\title{
Exploring the space of embedded minimal surfaces of finite total curvature
}

\author{
Martin Traizet \\ May 7, 2007
}

\section{Introduction}

This paper investigates the space of embedded minimal surfaces of finite total curvature (FTC) in euclidean space. For a long time the only known examples were the plane and the catenoid. In 1982, C. Costa discovered a genus one example with 3 ends [1]. D. Hoffman and W. Meeks proved that the Costa surface is embedded and constructed, for each genus $\geq 1$, a one-parameter family of embedded FTC minimal surfaces with three ends [4]. Since then, several general methods to construct examples have been proposed [8], [5].

In [6], the author developed a construction which does not rely on symmetries as do the previous ones. The input data for this construction is a finite collection of points in the complex planes (the configuration), satisfying a set of algebraic equations (the balancing condition). The output is a family of embedded FTC minimal surfaces, whose geometry can be described quite explicitely from the configuration. Roughly speaking, the surface is made of planes with small catenoidal necks between them, and the configuration gives the position of the necks, see figure 1 .

There are two aspects of this construction which can be explored numerically. The first one is the search for balanced configurations. Most of the interesting examples I have found were discovered numerically. Sometime they can be fully understood mathematically. They provide numerical, or 
mathematical answer to several interesting questions about embedded FTC minimal surfaces.

The other aspect is pictures. How can we actually compute these minimal surfaces ? Theoretically, they are constructed using Weierstrass Representation. The Riemann surface is defined by opening nodes, a perfectly explicit construction. Then three holomorphic 1-forms $\phi_{1}, \phi_{2}, \phi_{3}$ (the Weierstrass data) are defined abstractly by prescribing periods. The question is : how can we compute numerically these holomorphic 1-forms ? We provide a constructive answer to this problem when all parts of the noded Riemann surface have genus zero, and explain how this can be used to implement the construction in [6].

Credits : the Maple software was used to carry all numerical computations described in this paper. The domains were triangulated using Mathlab. I would like to thank C. Georgelin for her help in using this package. The Maple files are available on my web page : http: //www. lmpt . univ-tours.fr/ ${ }^{\sim}$ traizet. Also, on this page the reader will find $3 \mathrm{D}$ versions of the pictures in this paper which can be freely rotated with the mouse.

\section{Balanced configurations}

A configuration is a collection of points $\left\{p_{k, i}: 1 \leq k \leq M, 1 \leq i \leq N_{k}\right\}$ in the complex plane, together with some positive real numbers $c_{1}, \cdots, c_{M}$. The points $p_{k, 1}, \cdots, p_{k, N_{k}}$ form the $k^{\text {th }}$ layer of points. There are $M$ layers, and $N_{k}$ points in the $k^{\text {th }}$ layer. (We also say that the points in the $k^{\text {th }}$ layer are the points at level $k$.) The total number of points is $N=N_{1}+\cdots+N_{M}$. The numbers $c_{1}, \cdots, c_{M}$ are called the neck-sizes. The type of the configuration is the sequence $N_{1}, \cdots, N_{M}$.

Given a configuration which is balanced and non-degenerate (we will explain these terms shortly), the output of Theorem 1 in [6] is a one parameter family of FTC minimal surfaces $\left\{\mathcal{M}_{s}\right\}_{0<s<\varepsilon}(\varepsilon$ small enough). These surfaces have genus $N-M$ and have $M+1$ embedded ends. They can be described geometrically as $M+1$ horizontal planes with small catenoidal necks between them, see figure 1 . There are $M$ layers of necks and $N_{k}$ necks in the $k^{\text {th }}$ layer, whose positions are given by the points $p_{k, 1}, \cdots, p_{k, N_{k}}$. The necks in the $k^{\text {th }}$ layer have waist radius $s c_{k}$. This geometric description only holds asymptotically when $s \rightarrow 0$. 

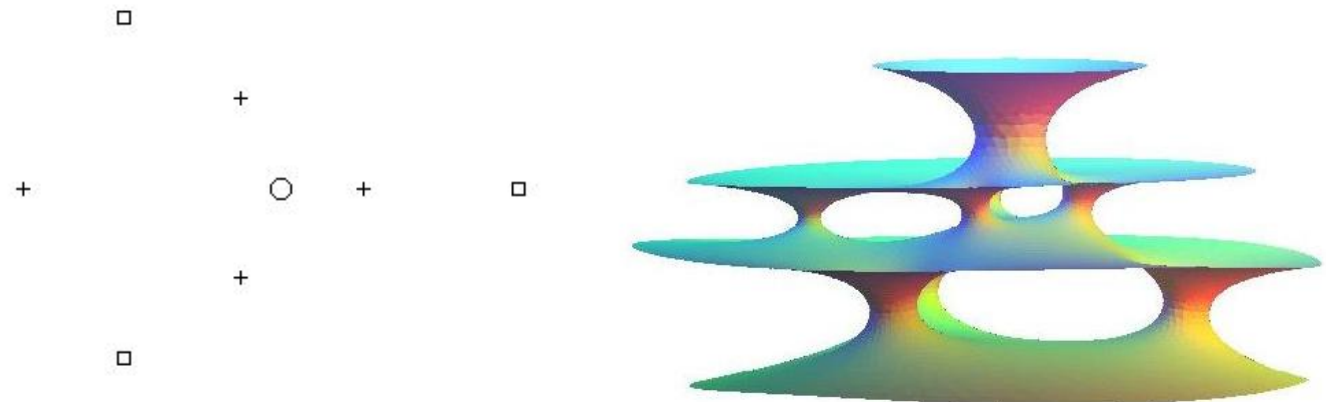

Figure 1: A configuration of type 3,4,1, and a genus 5 embedded minimal surface in the corresponding family. The boxes, crosses and circles represent the points at level 1,2 and 3 respectively.

The planes are perturbed to have logarithmic growth at infinity : the surface has $M+1$ catenoid type ends, whose logarithmic growths are as follows : let $Q_{k}=N_{k-1} c_{k-1}-N_{k} c_{k}$ (with the convention $N_{0}=N_{M+1}=$ $0)$. Then the logarithmic growth of the $k^{\text {th }}$ end is precisely $s Q_{k}$ for $k=$ $1, \cdots, M-1$, and is asymptotically $s Q_{k}$ for $k=M, M+1$ when $s \rightarrow 0$. (When the logarithmic growth is zero, the end is asymptotic to a plane). The theorem also guarantees that provided $Q_{1} \leq \cdots \leq Q_{M-1}<Q_{M}<Q_{M+1}$, the surfaces $\mathcal{M}_{s}$ are embedded (for $s$ small enough). When this condition is satisfied, we say that the configuration is embedded (a rather clumsy, but convenient terminology).

Let me now explain the balancing condition. Let

$$
F_{k, i}=\sum_{\substack{j=1 \\ j \neq i}}^{N_{k}} \frac{2 c_{k}^{2}}{p_{k, i}-p_{k, j}}-\sum_{j=1}^{N_{k-1}} \frac{c_{k} c_{k-1}}{p_{k, i}-p_{k-1, j}}-\sum_{j=1}^{N_{k+1}} \frac{c_{k} c_{k+1}}{p_{k, i}-p_{k+1, j}}
$$

with the convention that $N_{0}=N_{M+1}=0$. Because of the analogy with 2D electrostatic forces, we call $F_{k, i}$ the force on $p_{k, i}$. It interacts with all other points in the same layer, and with the points in the layer directly below and above it. We require that the points in each layer are distinct, and are distinct from the points in the layer directly below and above it, so that $F_{k, i}$ is defined. (We say the configuration is non-singular). 


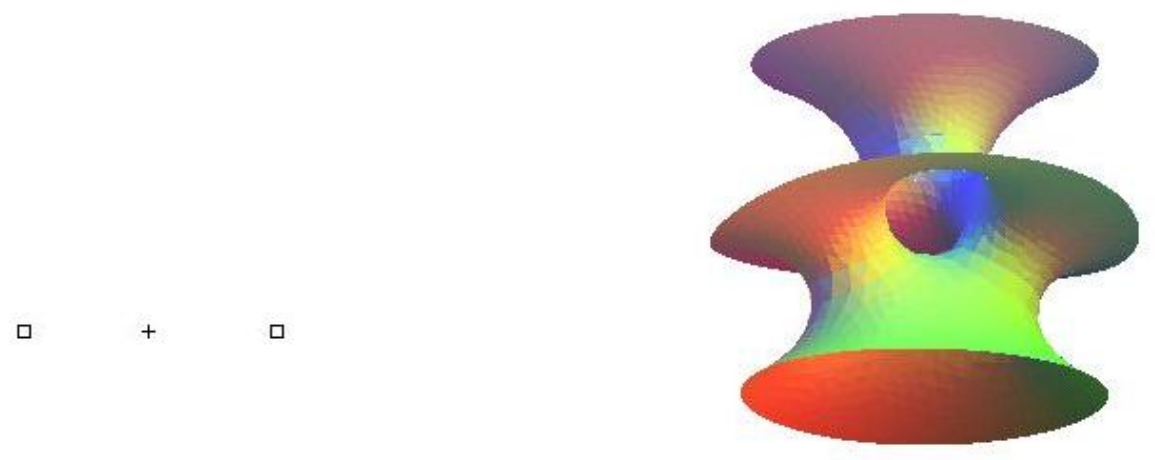

Figure 2: A genus one Costa Hoffman Meeks surface, as computed from the configuration on the left.

The configuration is balanced provided all forces are zero : $\forall k, \forall i, F_{k, i}=$ 0 . These are $N$ algebraic equations. These equations are not independent because one has $\sum_{k, i} F_{k, i}=0$. Moreover,

$$
\sum_{k, i} p_{k, i} F_{k, i}=\sum_{k=1}^{M} n_{k}\left(n_{k}-1\right) c_{k}^{2}-\sum_{k=1}^{M-1} n_{k} n_{k+1} c_{k} c_{k+1}
$$

The right hand side only depends on the neck-sizes, and it must be equal to zero for balanced configurations to exist. Provided the neck-sizes satisfy this condition, we are left with $N-2$ equations to solve. The balancing condition is also invariant by translation and complex scaling of the points (transformations $z \rightarrow a z+b$ ). We may normalize the positions of two points, and are left with $N-2$ parameters.

We say the configuration is non-degenerate if the jacobian matrix $\partial F_{k, i} / \partial p_{\ell, j}$ has complex rank $N-2$. This is the maximum rank it may have.

\subsection{Basic example : Costa Hoffman Meeks}

The simplest examples have $M=2$ layers of necks, with $N_{1}=n \geq 2$ and $N_{2}=1$. The neck-sizes are $c_{1}=1, c_{2}=n-1$. The configuration has dihedral symmetry of order $n$ and is given by $p_{1, i}=\omega^{i}$ and $p_{2,1}=0$, where $\omega=\exp (2 \pi \mathrm{i} / n)$. It is non-degenerate, see details in [6], proposition 1 . The 
corresponding family of embedded FTC minimal surfaces is the Costa Hoffman Meeks family of genus $n-1$, or rather, the extreme part of this family. (I have no proof of this claim, except in the genus one case, by the classification in [2]. All I really know is that the surfaces have the same symmetries as the Costa Hoffman Meeks surfaces). These are also the only non-degenerate balanced configurations with 2 layers of necks, see [6], proposition 1.

\subsection{Dihedral configurations}

In this section we investigate the following question :

What is the least genus for an embedded, FTC minimal surface with $r \geq 2$ ends?

N. Kapouleas has proven the existence of embedded, FTC minimal surfaces with any number of ends [5]. His examples are constructed by desingularization of a finite set of co-axial catenoids and horizontal planes. However, the genus of his examples is very large by construction (in fact, it seems hard to even estimate the genus).

D. Hoffman and W. Meeks have conjectured that the answer to the above question is $r-2$. M. Weber and M. Wolf have constructed, for each $r \geq 4$, a FTC minimal surface with genus $r-2$ and $r$ ends [8]. However, they cannot mathematically prove that their examples are embedded, although numerical pictures seem to indicate that they are (at least for a few values of $r$ !).

Our result lies in between : we can prove existence of embbeded FTC minimal surfaces with arbitrary number of ends, with an estimate of the genus, but this estimate is not optimal.

The easiest way to compute balanced configurations with arbitrary number of layers $M$ is to keep the symmetries of the Costa Hoffman Meeks configuration and increase the number of layers (see figure 3). Let $M \geq 2$ be the number of layers. Take $N_{1}=\cdots=N_{M-1}=n$ and $N_{M}=1$, where $n \geq 2$ is some integer. We want the configuration to have dihedral symmetry of order $n$, so we set

$$
p_{k, i}=a_{k} \omega^{i}, \quad 1 \leq k \leq M-1, \quad p_{M, 1}=0
$$

where $\omega=\exp (2 \pi \mathrm{i} / n)$ and $a_{k}$ is such that $a_{k}^{n} \in \mathbb{R}^{*}$. Equation (1) gives

$$
\sum_{k=1}^{M-1} n(n-1) c_{k}^{2}-\sum_{k=1}^{M-2} n^{2} c_{k} c_{k+1}=n c_{M-1} c_{M} .
$$


$\square$

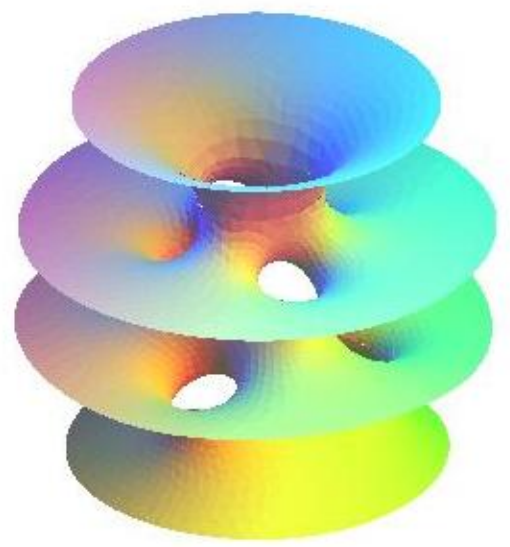

Figure 3: A configuration of type 3,3,1 with dihedral symmetry, and a genus 4 embedded minimal surface in the corresponding family.

This determines $c_{M}$ as a function of the parameters $c_{1}, \cdots, c_{M-1}$. By symmetry $p_{M, 1}=0$ and $p_{k, i} F_{k, i}$ is the same for all $i$. In fact, elementary computations give

$$
p_{1, i} F_{1, i}=(n-1) c_{1}^{2}-n c_{1} c_{2} \frac{a_{1}^{n}}{a_{1}^{n}-a_{2}^{n}}
$$

and for $2 \leq k \leq M-2$,

$$
p_{k, i} F_{k, i}=(n-1) c_{k}^{2}-n c_{k-1} c_{k} \frac{a_{k}^{n}}{a_{k}^{n}-a_{k-1}^{n}}-n c_{k} c_{k+1} \frac{a_{k}^{n}}{a_{k}^{n}-a_{k+1}^{n}} .
$$

We can fix $a_{1}=1$, then these equations determine recursively $a_{2}, \cdots, a_{M-1}$ as functions of $c_{1}, \cdots, c_{M-1}$. The equation $p_{M-1, i} F_{M-1, i}=0$ is then automatically satisfied since $\sum p_{k, i} F_{k, i}=0$. Alternately, we can choose the values of $a_{1}, \cdots, a_{M-1}$, and the equations determine the value of $c_{1}, \cdots, c_{M-1}$.

For $M=2$ we recover the Costa Hoffman Meeks configuration. It turns out that if $M \geq 3$, these configurations are not always non-degenerate, but the following is true: for generic values of the parameters $c_{1}, \cdots, c_{M-1}$, the configuration is non-degenerate. Here generic means : outside the zero set of a non-zero polynomial. Indeed, non-degeneracy can be written as a polynomial equation in $c_{1}, \cdots, c_{M-1}$. To prove the statement, it suffices to prove that this polynomial is not identically zero, so it suffices to exhibit one 
set of values of the parameters such that the configuration is non-degenerate, for each $n$ and $M$. We give the details of this computation in appendix A.1. It is clear that for generic values of $c_{1}, \cdots, c_{M-1}$, the configuration is nonsingular, which in this case means that all $a_{k}$ are non-zero and $a_{k} \neq a_{k+1}$.

It remains to choose the neck-sizes such that the configuration is embedded. Take $c_{1}=\cdots=c_{M-1}=1$. Equation (2) gives $c_{M}=n-M+1$. Then $Q_{1}=-n, Q_{2}=\cdots=Q_{M-1}=0, Q_{M}=M-1$ and $Q_{M+1}=n-M+1$. The condition $Q_{M+1}>Q_{M}$ gives $n>2(M-1)$, so we can take $n=2 M-1$. We obtain a family of embedded minimal surface, whose genus is $2(M-1)^{2}$ and number of ends is $M+1$. This gives

Theorem 1 For each $r \geq 3$, there exists an embedded FTC minimal surface with $r$ ends and genus $2(r-2)^{2}$.

This estimate is by no mean optimal since the genus grows quadratically with $r$, whereas the Hoffman Meeks conjecture asks for linear growth. It is possible to improve this estimate, but not very much. In fact, it is possible to prove that in general, for a configuration with $M$ layers, if the total number of necks is less than $M(M-1) / 2$, the configuration cannot be embedded, whatever the repartition of the necks and the neck-sizes. Hence one cannot construct minimal surfaces with $r$ ends and genus less than $(r-1)(r-2) / 2$ with this approach : quadratic growth of the genus cannot be avoided.

I believe that the Hoffman conjecture is true, and that there exists families of examples of FTC minimal surfaces with $r$ ends and genus $r-2$, but the family does not remain embedded all the way : it stops to be embedded a long time before it eventually degenerates and enters the range of application of our construction.

\subsection{Asymmetrical configurations}

In this section we investigate the following question :

What is the least genus for an embedded FTC minimal surface with no symmetries?

By a symmetry, I mean an ambient isometry preserving the surface (other than the identity). In [6], an embedded, asymmetrical example of genus 45 with 5 ends was proven to exist, as well as examples of arbitrary high genus. 


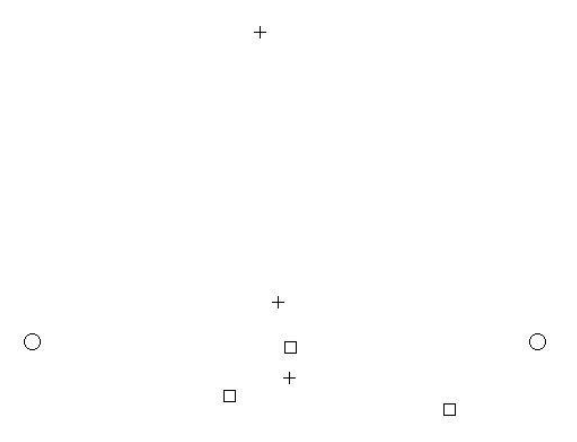

Figure 4: An asymmetrical configuration of type 3,4,2 (embedded, genus 6).

Numerical search gives plenty of embedded, asymmetrical examples of much smaller genus. The smallest genus I have found so far is 6 (see figure 4). Also, this numerical investigation has uncovered a genus 7 example which is simple enough so that it can be proven mathematically to exist (see figure 6 ). In this section, we explain how one can compute configurations without the help of symmetries, a situation quite opposite to section 2.2. A proof for the genus 7 example is given in appendix A.2.

The only non-degenerate configurations with $M=2$ layers are the Costa Hoffman Meeks configurations. The simplest next case is $M=3$ with $N_{3}=1$, which already gives plenty of interesting examples. Let me explain how I compute examples in this particular case. The method generalizes to arbitrary number of layers and necks, but is especially successful in this case.

Note that the balancing condition is invariant by permutation of the points at each level, so we should not use $p_{k, i}$ as variables when computing configurations, else each configuration will be duplicated $N_{1} ! N_{2}$ ! times, so the list of configurations will be huge and in fact the system will be impossible to solve. So the right variables are the symmetrical functions of the points at each level. Consider the polynomials

$$
P_{k}(z)=\prod_{i=1}^{N_{k}}\left(z-p_{k, i}\right) .
$$




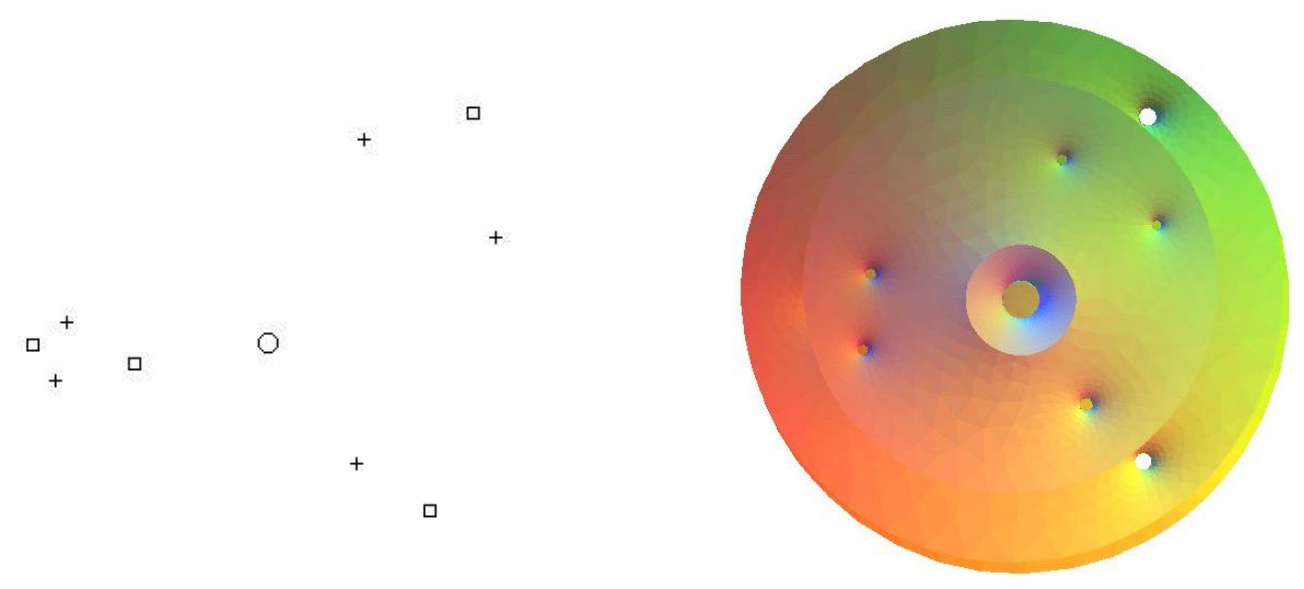

Figure 5: A genus 7 embedded, asymmetrical minimal surface, computed from a configuration of type $4,5,1$.

By translation, we may assume that $a_{3,1}=0$, so $P_{3}=z$. Let us write the forces in terms of $P_{1}, P_{2}$. We have

$$
\begin{gathered}
\frac{P_{k}^{\prime}}{P_{k}}(z)=\sum_{i} \frac{1}{z-p_{i}} \quad \text { if } P_{k}(z) \neq 0 . \\
\frac{P_{k}^{\prime \prime}}{P_{k}^{\prime}}\left(p_{k, i}\right)=\sum_{j \neq i} \frac{2}{p_{k, i}-p_{k, j}} . \\
F_{k, i}=c_{k}^{2} \frac{P_{k}^{\prime \prime}}{P_{k}^{\prime}}-c_{k} c_{k-1} \frac{P_{k-1}^{\prime}}{P_{k-1}}-c_{k} c_{k+1} \frac{P_{k+1}^{\prime}}{P_{k+1}} \quad \text { evaluated at } z=p_{k, i} .
\end{gathered}
$$

From this we get that the configuration is balanced if the polynomial

$$
c_{1}^{2} z P_{1}^{\prime \prime} P_{2}+c_{2}^{2} z P_{2}^{\prime \prime} P_{1}-c_{1} c_{2} z P_{1}^{\prime} P_{2}^{\prime}-c_{2} c_{3} P_{1} P_{2}^{\prime}
$$

vanishes at the points $p_{1,1}, \cdots, p_{1, N_{1}}$ and $p_{2,1}, \cdots, p_{2, N_{2}}$. Since this polynomial has degree $\leq N_{1}+N_{2}-1$, it is identically zero. Writing that all coefficients of this polynomial are zero gives a system of $N_{1}+N_{2}$ quadratic equations in the coefficients of $P_{1}, P_{2}$. The leading coefficient of (5) is

$$
N_{1}\left(N_{1}-1\right) c_{1}^{2}+N_{2}\left(N_{2}-1\right) c_{2}^{2}-N_{1} N_{2} c_{1} c_{2}-N_{2} c_{2} c_{3}
$$


so we recover equation (1). We are left with $N_{1}+N_{2}-1$ equations to solve. The parameters are the $N_{1}+N_{2}$ coefficients of $P_{1}$ and $P_{2}$. We may fix the value of one coefficient by complex scaling of the configuration.

This system has a special form which makes it easier to solve. We may use the first $N_{1}$ equations to express the coefficients of $P_{1}$ as functions of the coefficients of $P_{2}$, solving a linear system of $N_{1}$ equations. By substitution in the last $N_{2}-1$ equations, we obtain a system of algebraic equations in the $N_{2}-1$ coefficients of $P_{2}$ (one coefficient of $P_{2}$ is normalized by scaling). This works fairly well if $N_{2}$ is small. If $N_{1}$ is small, we can exchange the roles of $N_{1}$ and $N_{2}$.

Once we have found a couple of polynomials $P_{1}, P_{2}$ satisfying (5), we recover a configuration by computing their zeroes $p_{1,1}, \cdots, p_{1, N_{1}}$ and $p_{2,1}, \cdots, p_{2, N_{2}}$. We still must check that the configuration is non-singular, in the sense that these points are distinct, so the forces are defined. In fact, (5) always has the trivial solution $P_{k}=z^{N_{k}}$, which gives a useless configuration where all points are equal to 0 . But are the other ones non-singular?

Note that the balancing condition does not make sense for a singular configuration, but equation (5) still does, and may be seen as a way to make sense of a balanced singular configuration. In fact, families of configuration typically become singular for some particular values of the neck-sizes. But in the present case, this can happen only for a finite number of values. To understand why, assume that $z_{0}$ is a zero of $P_{k}$ with multiplicity $m_{k} \leq N_{k}$, for $k=1,2,3$ (if $m_{3}=1$ then $z_{0}=0$ ). Assume that $m_{1}+m_{2} \geq 2$ or $m_{2}+$ $m_{3} \geq 2$, so the configuration is singular. Also assume that $\left(m_{1}, m_{2}, m_{3}\right) \neq$ $\left(N_{1}, N_{2}, N_{3}\right)$, else $P_{k}=z^{N_{k}}$ for $k=1,2,3$ and we have the trivial solution to (5). Writing $P_{k}=\lambda_{k}\left(z-z_{0}\right)^{m_{k}}+o\left(\left(z-z_{0}\right)^{m_{k}}\right)$ and replacing in (5), we obtain the equation

$$
m_{1}\left(m_{1}-1\right) c_{1}^{2}+m_{2}\left(m_{2}-1\right) c_{2}^{2}-m_{1} m_{2} c_{1} c_{2}-m_{2} m_{3} c_{2} c_{3}=0 .
$$

Eliminating $c_{3}$ from (6) and (7) we obtain a quadratic homogenous equation in the unknowns $c_{1}, c_{2}$, whose coefficients depend on $\left(m_{1}, m_{2}, m_{3}\right)$. By inspection, we find that the coefficients of this equation are not all zero, so normalizing by $c_{1}=1$, there are at most two possible values for $c_{2}$. Since there are only a finite possible number of values for the triple $\left(m_{1}, m_{2}, m_{3}\right)$, there are only a finite number of values of $c_{2}$ for which a singular configuration can occur. 


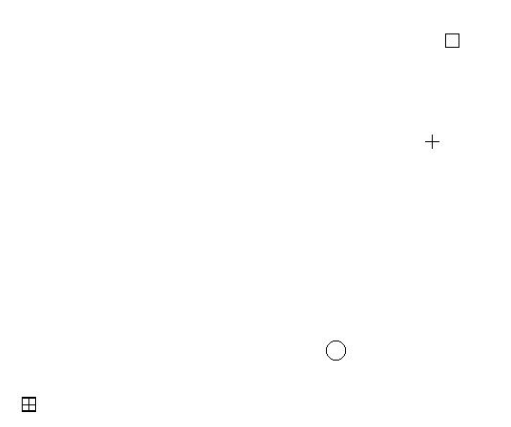

Figure 6: An asymmetrical configuration of type 5,4,1. There is a cluster of 4 points on the left and a cluster of 3 points on the right. This configuration is almost singular.

Figure 6 displays a configuration of type 5,4,1 with $c_{2}=1.01$. This configuration is almost singular, it becomes singular when $c_{2}=1$. One can compute the configuration quite explicitely when $c_{2}=1$, and conclude that it is asymmetrical. The details of this computation are given in appendix A.2. This proves the existence of an embedded, asymmetrical FTC minimal surface of genus 7 .

\subsection{A minimal surface with a planar end of order 2}

In this section we investigate the following question :

Can an embedded FTC minimal surface have a planar end of order two?

We are talking about the order of the extended Gauss map at the puncture corresponding to the end. This order is always at least 2 for a planar end. There are examples of periodic minimal surfaces with planar ends of order 2, like the Riemann minimal examples. For the previously known examples of FTC minimal surfaces with planar ends, the order of the Gauss map at the end was always at least 3. But this was in fact forced by the symmetries of these surfaces. In this section we exhibit an example with a planar end of order 2 .

We consider a configuration of type $5,4,1$. We take $c_{1}=1$ and $c_{2}=5 / 4$, equation (1) gives $c_{3}=11 / 4$. The logarithmic growths of the ends are $Q_{1}=$ 
$-5, Q_{2}=0, Q_{3}=9 / 4, Q_{4}=11 / 4$, so the end at level 2 is planar.

The question is, how can we determine the order of the Gauss map at the planar end ? Theoretically, we have the following asymptotic for the Gauss map $g_{s}$ of $\mathcal{M}_{s}$ in a neighborhood of the planar end (corresponding to $z=\infty$ )

$$
\lim _{s \rightarrow 0}-2\left(s g_{s}(z)^{-1}\right)=\sum_{i=1}^{N_{1}} \frac{c_{1}}{z-p_{1, i}}-\sum_{i=1}^{N_{2}} \frac{c_{2}}{z-p_{2, i}} .
$$

The right hand side can be expanded as

$$
\left(N_{1} c_{1}-N_{2} c_{2}\right) z^{-1}+\left(c_{1} \sum_{i=1}^{N_{1}} p_{1, i}-c_{2} \sum_{i=1}^{N_{2}} p_{2, i}\right) z^{-2}+o\left(z^{-2}\right) .
$$

The first term vanishes because the end is planar. The Gauss map has order 2 if the second term is not zero. This condition is easy to check.

The configuration can be computed using the methods of section 2.3. Unfortunately, I was not able to prove mathematically (meaning by hand) that this configuration is non-degenerate. (What I can prove is that the configuration is non-degenerate for generic values of the neck-sizes, but here the neck-sizes are fixed.)

The computation can easily be done with a formal computer, however. Moreover, the computation only involves rational numbers so can be carried using exact arithmetic. This gives a numerical proof that there exists embedded FTC minimal surface with a planar end of order 2. The details of this computation are given in appendix A.3.

\section{Pictures}

The goal of this section is to explain how, given a balanced configuration, one can compute numerically the corresponding family of minimal surfaces. This is illustrated on figure 7 in the case of the Costa Hoffman Meeks genus one family, corresponding to a configuration of type 2,1. The surface is decomposed into three pieces, one per end. Each piece is parametrized on a canonical circle domain, namely, the complex plane minus one or several round disks. The point at infinity correspond to an end of the surface. (In practice we clip the ends, so each piece is parametrized on a big disks minus small disks.) 

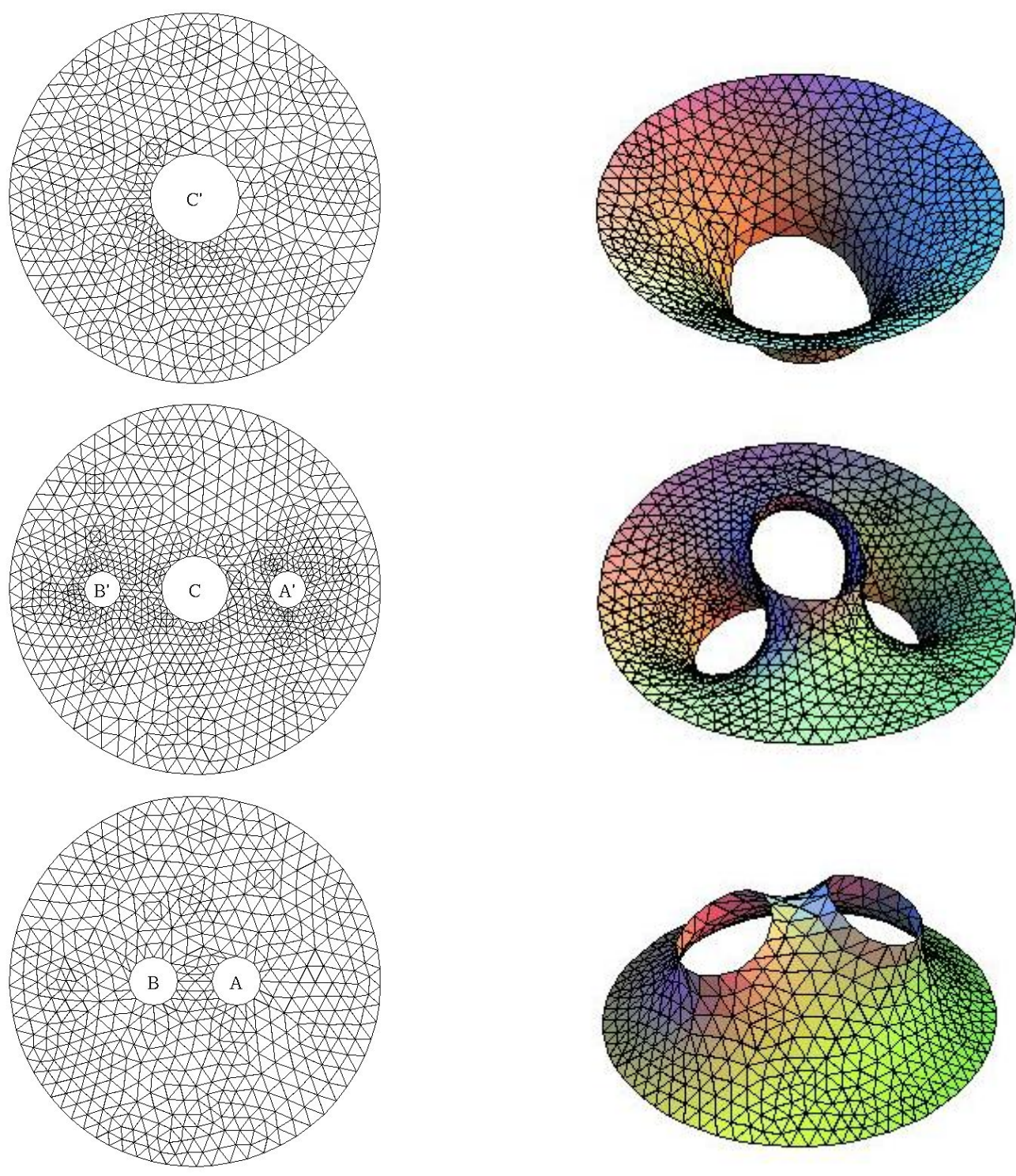

Figure 7: Triangulation of the three circle domains generated with Mathlab (left) and their image in space (right), in the case of the Costa Hoffman Meeks genus one surface. The three pieces on the right match perfectly to give the whole surface. 
If we identify the circle marked $A$ with the circle marked $A^{\prime}$, the circle marked $B$ with the circle marked $B^{\prime}$ and the circle marked $C$ with the circle marked $C^{\prime}$, we obtain topologically a genus one surface with three ends. This is our point of view on Riemann surfaces : canonical circles domains, with pairs of circles identified. This kind of construction is called opening nodes, because in the limit when the radii of the circles go to zero, we get a singular Riemann surface with nodes (or double points).

The minimal surface is parametrized by the Weierstrass Representation in the following form :

$$
X(z)=\left(\operatorname{Re} \int_{z_{0}}^{z} \phi_{1}, \operatorname{Re} \int_{z_{0}}^{z} \phi_{2}, \operatorname{Re} \int_{z_{0}}^{z} \phi_{3}\right)
$$

where $\phi_{1}, \phi_{2}$ and $\phi_{3}$ are three holomorphic 1-forms on our Riemann surface, such that $\phi_{1}^{2}+\phi_{2}^{2}+\phi_{3}^{2}=0$ (the conformality equation). The Period Problem asks that $X(z)$ is well defined (independent of the integration path).

To find three holorphic 1-forms satisfying the conformality equation and the Period Problem, we follow the following strategy : we construct $\phi_{1}, \phi_{2}, \phi_{3}$ by prescribing their periods around the necks. (These periods are imaginary, so part of the Period Problem is already solved by definition. Each imaginary part is a parameter). Then we adjust the parameters to solve $\phi_{1}^{2}+\phi_{2}^{2}+\phi_{3}^{2}=0$ and the remaining Period Problem.

This strategy has been used in [7] to construct triply periodic minimal surfaces. We follow the construction in [7], with a few modifications due to the fact that our minimal surfaces have ends.

This is not the strategy used to construct the family of minimal surfaces in [6], where we use the classical form of the Weierstrass Representation (with the Gauss map). The construction in [6] seems more difficult to implement.

The main question we have to answer to implement numerically the construction in [7] is : how can we compute numerically these holomorphic 1-forms ? For each one of them, we need some kind of formula, in each canonical circle domain. We answer this question in section 3.1. Each 1form is represented by some kind of series, whose coefficients are determined by solving a linear system.

At this point we have a family of Riemann surfaces, and holomorphic 1 -forms $\phi_{1}, \phi_{2}, \phi_{3}$, depending on a lot of parameters. These parameters are 
the center and radius of the circles, one Dehn twist parameter per neck, and the imaginary part of the periods around the necks.

We have a well defined immersion on each canonical circle domain, but there is no reason that the images fit together. For instance on figure 7 , the image of the circles $A$ and $A^{\prime}$ differ by a translation, and so do the images of the circles $B$ and $B^{\prime}$. But there is no reason that these translations are the same (as is the case on figure 7 ) : we need to adjust the parameters so that this is the case. This is the Period Problem.

We also have to adjust the parameters so that $\phi_{1}^{2}+\phi_{2}^{2}+\phi_{3}^{2}=0$, so that the immersion is minimal. Let $\psi=\phi_{1}^{2}+\phi_{2}^{2}+\phi_{3}^{2}$. This is a meromorphic quadratic differential with at most double poles at the ends. The space of such differentials has finite complex dimension $3 N-M-1$. The question is : how do we compute numbers from a quadratic differential ? Following the construction in [7], we simply compute periods of $\psi / d z$ along suitable cycles, and check that we obtain $3 N-M-1$ independent equations.

The total number of (real) equations we have to solve is $9 N-5 M-3$. This is a quite large number of equations : for example, for the asymmetrical example of figure 5 , with $N=10$ and $M=3$, we have 72 equations to solve. How do we solve such a large nonlinear system ?

Theoretically, in [7], we solve these equations using the Implicit Function Theorem. The proof of the Implicit Function Theorem, which is based on the contraction mapping principle, gives a method to numerically compute the solution, namely : assume we have a system of $n$ equations in $n$ variables, depending on some parameter $s$, which we write as $f_{s}(x)=0$. Assume that when $s=0$, we are given a solution $x_{0}$, and we know that $A=d f_{0}\left(x_{0}\right)$ is invertible. Then for $s$ small, we can solve $f_{s}(x)=0$ by the following iteration scheme : define the sequence $\left\{x^{k}\right\}_{k \in \mathbb{N}}$ inductively by $x^{0}=x_{0}$ and

$$
x^{k+1}=x^{k}-A^{-1} f_{s}\left(x^{k}\right) .
$$

Then for $s$ small, $\left\{x^{k}\right\}_{k \in \mathbb{N}}$ converges to a solution $x$ of $f_{s}(x)=0$.

We apply this scheme to solve our equations. In a few words : At the point $x_{0}$, the underlying Riemann surface is fully noded (all circles have radius zero : the necks collapse to double points), and the center of the circles are given by the points of the configuration. We can compute explicitely all equations at this point, see [7] for more details. The equations boil down to 
the balancing condition. Moreover, we can compute explicitely the jacobian matrix $A$ (derivative of equations with respect to parameters) at the point $x_{0}$. Provided the configuration is non-degenerate, the jacobian matrix is invertible. We then invert numerically the jacobian and apply the iteration scheme (8). For small values of $s$ it converges very quickly to a fixed point (several digits of precision are gained at each loop).

When we increase the value of $s$ it doesn't converge anymore. Here is the idea to push the parameter $s$ further : start from a previously computed solution $x_{s}$, increase $s$ by a small amount, and apply again the above iteration scheme with $x^{0}=x_{s}$ and $A$ equal to the jacobian at $x_{s}$. The basic problem is that as soon as $s$ is not zero, the nodes open (the circles have nonzero radius), so we cannot compute explicitely the jacobian matrix. However, we can compute a good approximation of the jacobian by pretending that the Riemann surface is still noded. Because the radii remain quite small (even though the other parameters move quite a lot), this gives us an approximate jacobian which we can use instead of $A$.

Finally, to plot the surface, we need to integrate $\phi_{1}, \phi_{2}, \phi_{3}$. Because these 1-forms are represented as series, their integrals are readily computed : no numerical integration is required.

The rest of the section is organized as follows : in section 3.1 we explain how we compute numerically a holomorphic 1-form defined by prescribing periods in the case of opening nodes. In section 3.2, we give more details about the construction of the family of minimal surfaces.

\subsection{Opening nodes : a model case}

In this section we explain how to compute holomorphic 1-forms on Riemann surfaces defined by opening nodes. For the simplicity of notations, we first consider the case where the noded Riemann surface has only one part, which has genus zero. Then we explain how one can generalize the construction to other cases.

Consider $2 N$ distinct points $a_{1}^{-}, \cdots, a_{N}^{-}, a_{1}^{+}, \cdots, a_{N}^{+}$in the complex plane We assume for convenience that the disks of radius one centered at these points are disjoint. Identify for each $i=1, \cdots, N$ the point $a_{i}^{-}$with the point $a_{i}^{+}$. This defines a noded Riemann surface $\Sigma_{0}$ with $N$ nodes, which we call $a_{1}, \cdots, a_{N}$. To open the nodes, consider $N$ complex numbers $t_{1}, \cdots, t_{N}$ 
such that $0<\left|t_{i}\right|<1$. Remove the $2 N$ disks $D\left(a_{i}^{ \pm},\left|t_{i}\right|\right)$. Identify the annulus $\left|t_{i}\right|<\left|z-a_{i}^{-}\right|<1$ with the annulus $\left|t_{i}\right|<\left|z^{\prime}-a_{i}^{+}\right|<1$ under the identification rule $\left(z-a_{i}^{-}\right)\left(z^{\prime}-a_{i}^{+}\right)=t_{i}$. This defines a Riemann surface $\Sigma_{t}$, where $t=\left(t_{1}, \cdots, t_{N}\right)$. We compactify $\Sigma_{t}$ by adding the point at infinity, and we still denote by $\Sigma_{t}$ the compactification. The genus of $\Sigma_{t}$ is $N$.

It is well known that the space of holomorphic 1-forms on $\Sigma_{t}$ has complex dimension $N$, and that a holomorphic 1-form is uniquely defined by prescribing its periods on the circles around the points $a_{1}^{+}, \cdots, a_{N}^{+}$. Let $\omega$ be the holomorphic 1-form on $\Sigma_{t}$ defined by prescribing

$$
\int_{C\left(a_{i}^{+}, 1\right)} \omega=2 \pi \mathrm{i} c_{i}, \quad i=1, \cdots, N
$$

where $c_{1}, \cdots, c_{N}$ are given complex numbers. The question is : how can we actually compute $\omega$ ?

We are aiming for a formula of the form

$$
\omega=\sum_{ \pm} \sum_{i=1}^{N} \sum_{n=1}^{\infty} \frac{A_{i, n}^{ \pm}}{\left(z-a_{i}^{ \pm}\right)^{n}} d z
$$

where the complex numbers $A_{i, n}^{ \pm}$are such that $A_{i, n}^{ \pm} t_{i}^{-n}$ is bounded, so that the series converges on the domain $\left|z-a_{i}^{ \pm}\right|>\left|t_{i}\right|$. (In the above formula, the sum on \pm means that we add two terms, on for + and one for -$)$. It is not hard to see that $\omega$ admits such a representation, using a Laurent series in the annuli $\left|t_{i}\right|<\left|z-a_{i}^{ \pm}\right|<1$ and the fact that a holomorphic 1-form on the Riemann sphere $\mathbb{C} \cup\{\infty\}$ must be identically zero.

The residues $A_{i, 1}^{ \pm}$are determined by the prescribed periods : since the circle $C\left(a_{i}^{+}, 1\right)$ is homologous in $\Sigma_{t}$ to the circle $C\left(a_{i}^{-}, 1\right)$ with the opposite orientation, we must have

$$
A_{i, 1}^{ \pm}= \pm c_{i}
$$

We want $\omega$ to be well defined on $\Sigma_{t}$, namely invariant under the identification rule used to define $\Sigma_{t}$. This should uniquely determine all coefficients $A_{i, n}^{ \pm}$. Let

$$
\varphi_{i}(z)=a_{i}^{-}+\frac{t_{i}}{z-a_{i}^{+}}
$$

so $\varphi_{i}$ maps the annulus $\left|t_{i}\right|<\left|z-a_{i}^{+}\right|<1$ to the annulus $\left|t_{i}\right|<\left|z-a_{i}^{-}\right|<1$ and $\Sigma_{t}$ is defined by identifying $z$ with $\varphi_{i}(z)$. The fact that $\omega$ is well defined 
on $\Sigma_{t}$ is equivalent to $\varphi_{i}^{*} \omega=\omega$ on the annulus $\left|t_{i}\right|<\left|\left(z-a_{i}^{+}\right)\right|<1$, for all $i=1, \cdots, N$. This is equivalent to

$$
\forall m \in \mathbb{Z}, \quad \int_{C\left(a_{i}^{+}, 1\right)}\left(z-a_{i}^{+}\right)^{m} \varphi_{i}^{*} \omega=\int_{C\left(a_{i}^{+}, 1\right)}\left(z-a_{i}^{+}\right)^{m} \omega
$$

By a change of variable,

$$
\int_{C\left(a_{i}^{+}, 1\right)}\left(z-a_{i}^{+}\right)^{m} \varphi_{i}^{*} \omega=-\int_{C\left(a_{i}^{-}, 1\right)}\left(\frac{t_{i}}{z-a_{i}^{-}}\right)^{m} \omega .
$$

So (10) may be rewritten as

$$
\forall m \geq 1, \quad A_{i, m+1}^{ \pm}=-\frac{t_{i}^{m}}{2 \pi \mathrm{i}} \int_{C\left(a_{i}^{\mp}, 1\right)} \frac{\omega}{\left(z-a_{i}^{\mp}\right)^{m}}
$$

(Notation : the sign $\mp$ on the right side is opposite to the sign \pm on the left side). This is an infinite dimensional linear system in the unknowns $A_{i, n}^{ \pm}$, $n \geq 2$. Let us introduce the following notations

$$
\begin{gathered}
\omega_{0}=\sum_{ \pm} \sum_{i=1}^{N} \frac{ \pm c_{i}}{z-a_{i}^{ \pm}} d z \\
A=\left(A_{i, n}^{ \pm}: i \leq N, n \geq 2\right), \\
\alpha(A)=\sum_{ \pm} \sum_{i=1}^{N} \sum_{n=2}^{\infty} \frac{A_{i, n}^{ \pm}}{\left(z-a_{i}^{ \pm}\right)^{n}} d z \\
F_{i, m}^{ \pm}(\alpha)=-\frac{t_{i}^{m-1}}{2 \pi \mathrm{i}} \int_{C\left(a_{i}^{\mp}, 1\right)} \frac{\alpha}{\left(z-a_{i}^{\mp}\right)^{m-1}}, \\
F(\alpha)=\left(F_{i, m}^{ \pm}(\alpha): i \leq N, m \geq 2\right) .
\end{gathered}
$$

Then (11) may be rewritten as $A=F\left(\omega_{0}+\alpha(A)\right)$. We solve this fixed point problem using the standard iteration scheme : define the sequence $\left\{A_{k}\right\}_{k \in \mathbb{N}}$ by induction by $A_{0}=0$ and $A_{k+1}=F\left(\omega_{0}+\alpha\left(A_{k}\right)\right)$. To see that $\left\{A_{k}\right\}_{k \in \mathbb{N}}$ converges to a fixed point, we introduce the following Banach norms :

$$
\|A\|=\sum_{ \pm} \sum_{i=1}^{N} \sum_{n=2}^{\infty}\left|A_{i, n}^{ \pm}\right|
$$




$$
\|\alpha\|_{\infty}=\sum_{ \pm} \sum_{i=1}^{N} \sup _{z \in C\left(a_{i}^{ \pm}, 1\right)}|\alpha(z)|
$$

Then straightforward estimates give

$$
\begin{gathered}
\|\alpha(A)\|_{\infty} \leq\|A\| \\
\|F(\alpha)\| \leq\left(\sum_{i=1}^{2 N} \frac{\left|t_{i}\right|}{1-\left|t_{i}\right|}\right)\|\alpha\|_{\infty}
\end{gathered}
$$

Hence $\left\|F\left(\omega_{0}\right)\right\|<\infty$ and provided all $t_{i}$ are small enough, $A \mapsto F(\alpha(A))$ is a contracting linear operator. It follows, by the standard fixed point theorem, that the sequence $\left\{A_{k}\right\}_{k}$ converges to a solution $A$ of $A=F\left(\omega_{0}+\alpha(A)\right)$. Let $\omega=\omega_{0}+\alpha(A)$. From (11), we have the estimate

$$
\left|A_{i, m}^{ \pm}\right| \leq\left|t_{i}\right|^{m-1}|| \omega \|_{\infty}
$$

Hence each series $\sum_{n} A_{i, n}^{ \pm}\left(z-a_{i}^{ \pm}\right)^{-n}$ converges for $\left|z-a_{i}^{ \pm}\right|>\left|t_{i}\right|$, so $\omega$ is the desired one form.

What we have achieved is a constructive proof of the existence and uniqueness of $\omega$ on $\Sigma_{t}$, provided all $t_{i}$ are small enough. The above method generalizes easily to the case of meromorphic differentials with prescribed principal part at the poles, provided the poles are outside the disks $D\left(a_{i}^{ \pm}, 1\right)$ : simply add the principal parts to $\omega_{0}$. The method also generalizes to the case of several Riemann spheres connected by nodes (see section 3.2), the notations are just a little more cumbersome. On the other hand, it is essential to the above argument that all the parts of the noded Riemann surface have genus zero so we can represent $\omega$ as a series.

There are several reasons why a constructive proof is interesting. First of all, this allows us to compute numerically $\omega$, which is our interest in this paper. The estimate (12) says that the coefficients $A_{i, n}^{ \pm}$decay rapidly with $n$, provided all $t_{i}$ remain small. So it is legitimate to truncate the series to some finite order. Typically for the examples we will consider, $\left|t_{i}\right|$ is of order 0.01 and we truncate the series to the order $n=10$.

The method is also interesting from the theoretical point of view because it generalizes to the case of infinitely many Riemann spheres connected by nodes. In this case, by opening the nodes we obtain non compact Riemann 
surfaces of infinite genus. The standard "abstract algebraic geometry" machinery does not seem to apply to this setup. This might be useful to construct, for instance, non-periodic minimal surfaces of infinite topology.

The integral in (11) can be explicitely computed using the following formula

$$
\operatorname{Res}_{b} \frac{1}{(z-a)^{n}(z-b)^{m}}=\left(\begin{array}{c}
n+m-2 \\
m-1
\end{array}\right) \frac{(-1)^{m-1}}{(b-a)^{n+m-1}}
$$

\subsection{How we compute the family of minimal surfaces}

In this section we give more details about how we compute numerically the family of minimal surfaces corresponding to a given configuration. As was already said, we follow very closely the construction in [7], with a few modifications to to the fact that our surfaces have catenoidal ends. In particular, the notations and normalizations are as in this paper. Giving all the details of the construction would essentially amount to write a proof of the main theorem in [6], which is not our point here, so we will be quite allusive.

We define a family of Riemann surfaces by opening nodes : We take $M+1$ copies of the complex plane, labeled $\mathbb{C}_{1}, \cdots, \mathbb{C}_{M+1}$. We take $N_{k}+$ $N_{k-1}$ distinct points $a_{k, 1}^{-}, \cdots, a_{k, N_{k}}^{-}$and $a_{k-1,1}^{+}, \cdots, a_{k-1, N_{k-1}}^{+}$in $\mathbb{C}_{k}$ (with the convention $\left.N_{0}=N_{M+1}=0\right)$. We identify the point $a_{k, i}^{-}$in $\mathbb{C}_{k}$ with the point $a_{k, i}^{+}$in $\mathbb{C}_{k+1}$, for each possible $(k, i)$. This defines a noded Riemann surface with $N=N_{1}+\cdots+N_{M}$ nodes.

We open the nodes as in section 3.1, using one complex parameter $t_{k, i}$ per node. This creates a neck connecting $\mathbb{C}_{k}$ with $\mathbb{C}_{k+1}$. We call $\Sigma_{t}$ the resulting Riemann surface, and $\infty_{1}, \cdots, \infty_{M+1}$ the points at infinity, which will correspond to the ends of our minimal surface. The genus of $\Sigma_{t}$ is $N-M$. (Note that the circle of center $a_{k, i}^{-}$in $\mathbb{C}_{k}$ and the circle of center $a_{k, i}^{+}$in $\mathbb{C}_{k+1}$, both of radius $\sqrt{\left|t_{i}\right|}$, are identified : these are the boundary circles of the canonical domains in figure 7 .)

Next we define three meromorphic 1-forms $\phi_{1}, \phi_{2}, \phi_{3}$ on $\Sigma_{t}$, with poles at $\infty_{1}, \cdots, \infty_{M+1}$, by prescribing periods on the circles $C\left(a_{k, i}^{+}, 1\right)$ as explained in section 3.1. The principal parts at the poles are forced by the fact that we want horizontal embedded ends : $\phi_{1}$ and $\phi_{2}$ need double poles, with no residue, and $\phi_{3}$ needs a simple pole. As explained in section 3.1, we represent 
$\phi_{\nu}$ as

$$
\phi_{\nu, k}=\lambda_{\nu, k} d z+\sum_{i=1}^{N_{k}} \sum_{n=1}^{\infty} \frac{A_{\nu, k, i, n}^{-}}{\left(z-a_{k, i}^{-}\right)^{n}} d z+\sum_{i=1}^{N_{k-1}} \sum_{n=1}^{\infty} \frac{A_{\nu, k-1, i, n}^{+}}{\left(z-a_{k-1, i}^{+}\right)^{n}} d z .
$$

Here $\phi_{\nu, k}$ denotes $\phi_{\nu}$ in $\mathbb{C}_{k}$. The first term takes care of the double pole at $\infty_{k}$. We require that

$$
\lambda_{1, k}=1, \quad \lambda_{2, k}=(-1)^{k+1} \mathrm{i}, \quad \lambda_{3, k}=0 .
$$

The residues are determined by the period conditions :

$$
A_{\nu, k, i, 1}^{ \pm}= \pm c_{\nu, k, i}
$$

where $c_{\nu, k, i}$ are prescribed real numbers.

As explained in section 3.1, the coefficients $A_{\nu, k, i, n}$ for $n \geq 2$ may be computed by solving a linear system by iteration. Adapted to the case at hand, this gives the following recipe :

$$
\begin{aligned}
A_{\nu, k, i, m+1}^{ \pm} \leftarrow & -\delta_{m, 1} \lambda_{\nu, k} t_{k, i}+\left(-t_{k, i}\right)^{m} \sum_{n=1}^{\infty}\left(\begin{array}{c}
n+m-2 \\
m-1
\end{array}\right) \\
& {\left[\sum_{\substack{j=1 \\
j \neq i}}^{N_{k}} \frac{A_{\nu, k, j, n}^{\mp}}{\left(a_{k, i}^{\mp}-a_{k, j}^{\mp}\right)^{n+m-1}}+\sum_{j=1}^{N_{k \mp 1}} \frac{A_{\nu, k \mp 1, j, n}^{ \pm}}{\left(a_{k, i}^{\mp}-a_{k \mp 1, j}^{ \pm}\right)^{n+m-1}}\right] . }
\end{aligned}
$$

$\left(\delta_{i, j}\right.$ denotes the Kronecker symbol). Namely, we compute the right hand side for all $\nu, k, i$ and for all $m \geq 1$, we replace all $A_{\nu, k, i, m+1}$ by the values we have found, and we iterate this process until each $A_{\nu, k, i, m+1}$ is equal to the right hand side at the desired order of accuracy. As was already said, we also truncate the series to some reasonnable order, depending on how small the parameters $t_{k, i}$ are.

The meromorphic 1-forms can be explicitely integrated :

$$
\begin{aligned}
X_{\nu, k}(z)= & \operatorname{Re} \int \phi_{\nu, k} \\
= & \lambda_{\nu, k} z+\sum_{i=1}^{N_{k}}\left(A_{\nu, k, i, 1} \log \left|z-a_{k, i}^{-}\right|+\operatorname{Re} \sum_{n=2}^{\infty} \frac{A_{\nu, k, i, n}^{-}}{(1-n)\left(z-a_{k, i}^{-}\right)^{n-1}}\right) \\
& +\sum_{i=1}^{N_{k-1}}\left(A_{\nu, k-1, i, 1} \log \left|z-a_{k-1, i}^{+}\right|+\operatorname{Re} \sum_{n=2}^{\infty} \frac{A_{\nu, k-1, i, n}^{+}}{(1-n)\left(z-a_{k-1, i}^{+}\right)^{n-1}}\right) .
\end{aligned}
$$


The Period Problem can be written as

$$
X_{\nu, k}\left(a_{k, i}^{-}+\sqrt{t_{k, i}}\right)-X_{\nu, k+1}\left(a_{k, i}^{+}+\sqrt{t_{k, i}}\right) \text { is independent of } i .
$$

Let $\psi=\phi_{1}^{2}+\phi_{2}^{2}+\phi_{3}^{2}$. Following [7], we solve the following equations :

$$
\begin{gathered}
\int_{C\left(a_{k, i}^{-}, 1\right)}\left(z-a_{k, i}^{-}\right) \frac{\psi}{d z}=0, \quad 1 \leq k \leq M, \quad 1 \leq i \leq N_{k} \\
\int_{C\left(a_{k, i}^{+}, 1\right)} \frac{\psi}{d z}=0, \quad 1 \leq k \leq M, \quad 2 \leq i \leq N_{k} \\
\int_{C\left(a_{k, i}^{-}, 1\right)} \frac{\psi}{d z}=0, \quad 1 \leq k \leq M, \quad 1+2 \delta_{k, 1} \leq i \leq N_{k} \\
\operatorname{Im}\left(\sum_{i=1}^{2} a_{1, i}^{-} \int_{C\left(a_{1, i}^{-}, 1\right)} \frac{\psi}{d z}\right)=0
\end{gathered}
$$

Provided the Period Problem is solved (namely all periods of $\phi_{1}, \phi_{2}$ and $\phi_{3}$ are pure imaginary), $\psi$ also automatically satisfies the following equation

$$
\sum_{k=1}^{M+1}(-1)^{k}\left[\sum_{i=1}^{N_{k}} \int_{C\left(a_{k, i}^{-}, 1\right)} z \frac{\psi}{d z}+\sum_{i=1}^{N_{k-1}} \int_{C\left(a_{k-1, i}^{+}, 1\right)} z \frac{\psi}{d z}\right] \in \mathrm{i} \mathbb{R} .
$$

This mysterious relation is a consequence of Riemann's bilinear relation. For completeness we give a proof of this equation in appendix A.4. By the same argument as in [7], one can prove that provided $t$ is small enough, these $6 N-2 M-2$ real equations are linearly independent, so solving this system guarantees that $\psi=0$. For completeness, we provide a proof of this statement in appendix A.5.

\section{A Appendix}

\section{A.1 Dihedral configurations are non-degenerate}

In this section we prove that the dihedral configurations of section 2.2 are non-degenerate for generic values of the parameters $c_{1}, \cdots, c_{M-1}$. As explained in this section, it suffices to find one set of values of the parameters such that the configuration is non-degenerate.

We take $a_{k}=t^{2^{k}}$ for $1 \leq k \leq M-1$. We shall prove that for $t>0$ small enough, the configuration is non-degenerate. 
The idea is the following : scale the configuration so that the points at level $k$ are the $n$th roots of unity. Then the points at level $<k$ go to infinity and the points at level $>k$ go to 0 when $t \rightarrow 0$, and in the limit we get a Costa Hoffman Meeks configuration. So for small $t$, the configuration may be seen as several Costa Hoffman Meeks configuration imbricated into each other. Non-degeneracy thus boils down to the fact that the Costa Hoffman Meeks configuration is non-degenerate.

We may normalize $c_{1}=1$. Then $c_{2}, \cdots, c_{M-1}$ are determined inductively by (3), (4) and $C_{M}$ is determined by (2). Their limits when $t \rightarrow 0$ are

$$
\lim c_{k}=\left(\frac{n-1}{n}\right)^{k-1}
$$

for $1 \leq k \leq M-1$ and

$$
\lim c_{M}=\frac{(n-1)^{M-1}}{n^{M-2}} .
$$

Tedious computations give the following limits for the partial derivatives of the forces :

$$
\begin{gathered}
\lim \left(t^{2^{k+1}} \frac{\partial F_{k, i}}{\partial p_{k, i}}\right)=\lim c_{k}^{2}\left(\frac{n-1}{\omega^{2 i}}-2 \sum_{j \neq i} \frac{1}{\left(\omega^{i}-\omega^{j}\right)^{2}}\right) \\
\lim \left(t^{2^{k+1}} \frac{\partial F_{k, i}}{\partial p_{k, j}}\right)=\lim c_{k}^{2} \frac{2}{\left(\omega^{i}-\omega^{j}\right)^{2}} \quad \text { if } j \neq i . \\
\lim \left(t^{2^{k+1}} \frac{\partial F_{k, i}}{\partial p_{k-1, i}}\right)=0 . \\
\lim \left(t^{2^{k+1}} \frac{\partial F_{k, i}}{\partial p_{k+1, i}}\right)=-\frac{c_{k} c_{k+1}}{\omega^{2 i}} . \\
\left.\sum_{i} \omega^{i} \frac{\partial F_{k, i}}{\partial p_{k, j}}\right)=\lim c_{k} c_{k-1} \frac{n^{2}}{\omega^{j}} \quad \text { with the convention } c_{0}=0 . \\
\lim \left(t^{(4-n) 2^{k-1}} \sum_{i} \omega^{i} \frac{\partial F_{k, i}}{\partial p_{k-1, j}}\right)=0 . \\
\left.\sum_{i}^{(4-n) 2^{k-1}} \omega^{i} \frac{\partial F_{k, i}}{\partial p_{k+1, j}}\right)=-\delta_{2, n} \frac{n^{2} c_{k} c_{k+1}}{\omega^{j}} .
\end{gathered}
$$

Let $A$ be the $n \times n$ complex matrix defined by

$$
\begin{gathered}
A_{i, i}=\frac{n-1}{\omega^{2 i}}-2 \sum_{j \neq i} \frac{1}{\left(\omega^{i}-\omega^{j}\right)^{2}}, \\
A_{i, j}=\frac{2}{\left(\omega^{i}-\omega^{j}\right)^{2}} \quad \text { if } j \neq i .
\end{gathered}
$$


This is the jacobian matrix of the Costa Hoffman Meeks configuration, with the last row and column removed. Since the Costa Hoffman Meeks configuration is non-degenerate, $A$ has rank $n-1$ and any minor of size $n-1$ of $A$ is invertible. Let $B$ be the $n \times n$ matrix defined by $B_{i, j}=A_{i, j}$ for $i<n$ and $B_{n, j}=\omega^{-j}$. Then $B$ has rank $n$. Indeed, the operation $C_{n} \leftarrow \sum \omega^{j} C_{j}$ on the columns of $B$ gives the column $C_{n}=(0, \cdots, 0, n)$.

Returning to the dihedral configuration, put the variables in lexicographic order : $p_{1,1}, \cdots, p_{1, n}, \cdots, p_{M-1,1}, \cdots, p_{M-1, n}, p_{M, 1}$. Consider the jacobian matrix, remove the first line, the first column, the last line and the last column. Let $L_{k, i}$ denote the row corresponding to $F_{k, i}$. Perform the following row operations :

$$
\begin{gathered}
L_{k, 1} \leftarrow \frac{t^{(4-n) 2^{k-1}}}{n^{2} c_{k} c_{k-1}} \sum_{i} \omega^{i} L_{k, i} \quad \text { for } k \geq 2, \\
L_{k, i} \leftarrow \frac{t^{2^{k+1}}}{c_{k}^{2}} L_{k, i} \quad \text { for } k \geq 2, i \geq 2 .
\end{gathered}
$$

By the above formulae, one obtains a matrix which converges when $t \rightarrow 0$ to a matrix which has upper triangular block form, with $M$ square blocks on the diagonal. The first block has size $n-1$ and is an invertible minor of $A$. The other $M-1$ blocks are equal to $B$ so are invertible. Hence this limit matrix is invertible. It follows that the dihedral configuration is non-degenerate for $t$ small enough.

\section{A.2 An asymmetrical configuration of type 5, 4, 1 .}

In this section we give a proof that there exists a family of embedded asymmetrical configuration of type $5,4,1$, namely we take $N_{1}=5, N_{2}=4, N_{3}=1$. We may normalize $c_{1}=1 . c_{3}$ is determined in function of the free parameter $c_{2}$ by (6). By a straightforward computation, the configuration is embedded provided $1<c 2<\frac{5-\sqrt{5}}{2} \simeq 1.381$.

We first study the case $c_{2}=1$. Equation (6) gives $c_{3}=3$. Write

$$
P_{1}=z^{5}+\sum_{i=0}^{4} a_{i} z^{i}, \quad P_{2}=z^{4}+\sum_{i=0}^{3} b_{i} z^{i} .
$$

We assume that $a_{0} \neq 0$, as the case $a_{0}=0$ only gives very symmetric configurations. We also assume that $b_{3} \neq 0$, and take $b_{3}=2$ by scaling. Equation (5) with $z=0$ gives $b_{1}=0$. Expanding equation (5) we find

$$
\begin{aligned}
& 4\left(1-a_{4}\right) z^{7}-6\left(a_{3}+a_{4}-b_{2}\right) z^{6}-6\left(a_{2}+2 a_{3}\right) z^{5}-2\left(2 a_{1}+7 a_{2}-10 b_{0}+2 a_{3} b_{2}\right) z^{4} \\
& -6\left(2 a_{1}+a_{2} b_{2}-2 a_{4} b_{0}\right) z^{3}-6\left(a_{0}+a_{1} b_{2}-a_{3} b_{0}\right) z^{2}-\left(4 a_{0} b_{2}-2 a_{2} b_{0}\right) z=0 .
\end{aligned}
$$

Let $E_{i}$ be the coefficient of $z^{i}$ in this equation. Write $x=b_{2}$. Equations $E_{7}$ to $E_{2}$ in this order determine all coefficients as functions of $x$ by solving only linear equations. We find, in this order,

$$
a_{4}=1, \quad a_{3}=x-1, \quad a_{2}=2-2 x,
$$




$$
a_{0}=\frac{1}{4}\left(-4 x^{3}+2 x^{2}+9 x-7\right), \quad a_{1}=\frac{1}{4}\left(6 x^{2}-13 x+7\right), \quad b_{0}=\frac{1}{4}\left(2 x^{2}-9 x+7\right) .
$$

Reporting these values in $E_{1}$ gives the equation

$$
P(x):=4 x^{4}-4 x^{3}+2 x^{2}-9 x+7=0 .
$$

which factors as

$$
(x-1)\left(4 x^{3}+2 x-7\right)=0 .
$$

This polynomial has four simple roots, two of which are complex. Let $x_{0}$ be one of them. Using Euclid's algorithm we find that

$$
D:=\operatorname{gcd}\left(P_{1}, P_{2}\right)=z^{3}+\left(-x_{0}+2\right) z^{2}+\left(x_{0}^{2}-x_{0}\right) z+\frac{1}{4}\left(4 x_{0}^{2}+2 x_{0}-7\right)
$$

and $P_{1}, P_{2}$ factor as

$$
P_{1}=\left(z^{2}+\left(x_{0}-1\right) z-x_{0}+1\right) D, \quad P_{2}=\left(z+x_{0}\right) D .
$$

In particular $P_{1}$ and $P_{2}$ share three roots, so the configuration is singular. Let us prove that the configuration is asymmetrical if $x_{0}$ is not real. Let $\varphi: \mathbb{C} \rightarrow \mathbb{C}$ be a symmetry of the configuration (other than the identity), so $\varphi$ fixes the set of roots of $P_{k}$, for each $k=1,2,3$. Then $\varphi(0)=0$, and $\varphi$ fixes the sum of the roots of $P_{1}$, which is equal to 1. Hence $\varphi(z)=\bar{z}$. This implies that the coefficients of $P_{1}$ are real, hence $x_{0}$ is real, a contradiction. So the configuration is asymmetrical.

For arbitrary values of $c_{2}$, we can solve the equations in the same way, except that the computations cannot be explicitely done by hand, so we content ourself with the form of the solutions. In the following, the notation $\ell(\cdot)$ means a linear function of its arguments whose coefficients are rational functions of $c_{2}$. We normalize $b_{3}=1$ and write $b_{2}=x$ as before. Then (5) has the form

$$
\begin{aligned}
& \ell\left(a_{4}, 1\right) z^{7}+\ell\left(a_{3}, a_{4}, x\right) z^{6}+\ell\left(a_{2}, a_{3}, a_{4} x\right) z^{5}+\ell\left(a_{1}, a_{2}, a_{3} x, b_{0}\right) z^{4} \\
& +\ell\left(a_{0}, a_{1}, a_{2} x, a_{4} b_{0}\right) z^{3}+\ell\left(a_{0}, a_{1} x, a_{3} b_{0}\right) z^{2}+\ell\left(a_{0} x, a_{2} b_{0}\right) z=0
\end{aligned}
$$

Let $E_{i}$ be the coefficient of $z^{i}$ in this equation. Solving $E_{7}, E_{6}$ and $E_{5}$ gives

$$
a_{4}=p_{0}(x), \quad a_{3}=p_{1}(x), \quad a_{2}=p_{1}(x) .
$$

where the notation $p_{r}(x)$ denotes a polynomial of degree at most $r$ in the variable $x$ whose coefficients are rational functions of $c_{2}$. Equations $E_{4}, E_{2}$ and $E_{2}$ then give a linear system in the unknowns $a_{0}, a_{1}$ and $a_{2}$. The determinant $\Delta$ of this system has the form $\Delta=p_{1}(x)$. The Cramer formula gives

$$
a_{0}=\frac{p_{3}(x)}{\Delta}, \quad a_{1}=\frac{p_{3}(x)}{\Delta}, \quad b_{0}=\frac{p_{3}(x)}{\Delta} .
$$

Multiplying $E_{1}$ by Delta and replacing the above values gives an equation of the form $P(x)=0$, where $P$ is a polynomial of degree at most 4 whose coefficients are rational functions of $c_{2}$. When $c_{2}=1$, we have seen that that no division by zero occurs in this 
computation, and $P$ has four simple roots. Therefore, this remains true for generic values of $c_{2}$ (namely, except for a finite number of values). Also for $c_{2}=1, P$ has two complex roots. This remains true by continuity for $c_{2}$ close to 1 .

We have seen in section 2.3 that for generic values of $c_{2}$, the configuration is nonsingular. Let us now prove that it is non-degenerate, provided it is non-singular and $x_{0}$ is a simple root of $P$. Fix the values of $c_{1}, c_{2}$ and $c_{3}$. Let $p_{k, i}(t)$ be a deformation of the configuration with $p_{k, i}(0)=p_{k, i}$, where $t$ is a real parameter. Assume that $F_{k, i}(t)=$ $o(t)$. We must prove that up to complex scaling and translation, $p_{k, i}(t)=p_{k, i}(0)+o(t)$. Normalize translation by $p_{3,1}(t)=0$. Define as before the polynomials $P_{k, t}=\prod(z-$ $\left.p_{k, i}(t)\right)$. Equation (5) gives that

$$
c_{1}^{2} z P_{1, t}^{\prime \prime} P_{2, t}+c_{2}^{2} z P_{1, t} P_{2, t}^{\prime \prime}-c_{1} c_{2} z P_{1, t}^{\prime} P_{2, t}^{\prime}-c_{2} c_{3} P_{1, t} P_{2, t}^{\prime}=o(t)
$$

at the points $p_{1,1}(t), \cdots, p_{1, N_{1}}(t), p_{2,1}(t), \cdots, p_{2, N_{2}}(t)$. By linear algebra, the coefficients of the above polynomial are all $o(t)$. If we call $a_{0}(t), \cdots, a_{4}(t)$ and $b_{0}(t), \cdots, b_{3}(t)$ the coefficients of $P_{1, t}$ and $P_{2, t}$, normalize scaling by $b_{3}(t)=2$ and write $b_{2}(t)=x_{t}$, we obtain, by the above computation, the equation $P\left(x_{t}\right)=o(t)$, where $P$ is the same polynomial. (Recall that the coefficients of $P$ only depend $c_{2}$, which is fixed, so $P$ does not depend on t.) Since $x_{0}$ is a simple root of $P$, this implies that $x_{t}=x_{0}+o(t)$. Then we have $a_{i}(t)=$ $a_{i}(0)+o(t)$ and $b_{i}(t)=b_{i}(0)+o(t)$. Hence $p_{k, i}(t)=p_{k, i}(0)+o(t)$, so the configuration is non-degenerate.

We conclude that if $c_{2}>1$ is close enough to 1 , the configuration is non-singular, non-degenerate, asymmetric and embedded.

\section{A.3 An example with a planar end of order 2}

In this section, we give a computer proof that there exists embedded FTC minimal surfaces with a planar end of order 2 . We continue with the example of type $5,4,1$ of the previous section and take the value $c_{2}=5 / 4$, which gives a planar end at level 2. Here are the results :

$$
\begin{gathered}
a_{4}=\frac{5}{24}, \quad a_{3}=-\frac{105}{256}+\frac{15}{16} x, \quad a_{2}=\frac{3465}{2048}-\frac{4595}{1152} x \\
a_{0}=-\frac{1}{9437184} \frac{-493537968 x+121415679+768946176 x^{3}+162269440 x^{2}}{16 x+9} \\
a_{1}=\frac{1}{1966080} \frac{6967296 x^{3}-8351343+29794864 x-26814720 x^{2}}{16 x+9} \\
b_{0}=-\frac{49313}{18432} x+\frac{63}{256} x^{2}+\frac{71379}{65536} \\
P(x)=-1524209068800 x^{2}-285169111920 x+2183134638080 x^{3}+180302283315+1490178539520 x^{4} .
\end{gathered}
$$

The discriminant of $P$ is

137262067070756943236221183727217566386581834266359030391280500736000000 
which is non-zero, so $P$ has four simple roots. Each of them gives a non-degenerate configuration.

To prove that each configuration is non-singular, we use the argument described at the end of section 2.3. The only values of $\left(m_{1}, m_{2}, m_{3}\right)$ which satisfy (7), with $m_{1}+m_{2} \geq 2$ or $m_{2}+m_{3} \geq 2$, are $(1,4,1)$ and $(5,4,1)$. Both of them give $P_{2}=z^{4}$, which is not the case because $b_{3}=2$. So the configurations are non-singular. Finally, we have

$$
c_{1} \sum_{i} p_{1, i}-c_{2} \sum_{i} p_{2, i}=-c_{1} a_{4}+c_{2} b_{3}=\frac{55}{24} \neq 0
$$

so the Gauss map has order 2 at the planar end.

\section{A.4 Proof of equation (19)}

By the Residue theorem, we have

$$
\sum_{i=1}^{N_{k}} \int_{C\left(a_{k, i}^{-}, 1\right)} z \frac{\psi}{d z}+\sum_{i=1}^{N_{k-1}} \int_{C\left(a_{k-1, i}^{+}, 1\right)} z \frac{\psi}{d z}=-2 \pi \operatorname{ines}_{\infty_{k}}\left(z \frac{\psi}{d z}\right) .
$$

Let $g$ be the genus of $\Sigma$ and $A_{1}, \cdots, A_{g}, B_{1}, \cdots, B_{g}$ be a canonical homology basis of $\Sigma$. We apply Riemann's bilinear relation ([3], page 241) to the pair of meromorphic 1-forms $\left(\phi_{1}, \phi_{2}\right)$ (these are meromorphic differentials of the second kind)

$$
\sum_{i=1}^{g} \int_{A_{i}} \phi_{1} \int_{B_{i}} \phi_{2}-\int_{A_{i}} \phi_{2} \int_{B_{i}} \phi_{1}=2 \pi \mathrm{i} \sum_{k=1}^{M+1} \operatorname{Res}_{\infty_{k}}\left(\phi_{2} \int \phi_{1}\right) .
$$

By assumption, the period problem is solved, so all periods of $\phi_{1}$ and $\phi_{2}$ are imaginary. Hence the left side is real. To compute the residues at $\infty_{k}$, we write, in a neighborhood of $\infty_{k}$,

$$
\begin{gathered}
\phi_{1}=d z+\mu_{1, k} \frac{d z}{z^{2}}+o\left(\frac{d z}{z^{2}}\right), \\
\int \phi_{1}=z-\frac{\mu_{1, k}}{z}+o\left(\frac{1}{z}\right), \\
\phi_{2}=(-1)^{k+1} \mathrm{i} d z+\mu_{2, k} \frac{d z}{z^{2}}+o\left(\frac{d z}{z^{2}}\right), \\
\phi_{3}=Q_{k} \frac{d z}{z}+o\left(\frac{d z}{z}\right)
\end{gathered}
$$

where $\mu_{1, k}, \mu_{2, k}$ are some complex numbers and $Q_{k}$ is real. This gives

$$
\begin{gathered}
\operatorname{Res}_{\infty_{k}}\left(z \frac{\psi}{d z}\right)=-2 \mu_{1, k}+2(-1)^{k} \mathrm{i} \mu_{2, k}-Q_{k}^{2} \\
\operatorname{Res}_{\infty_{k}}\left(\phi_{2} \int \phi_{1}\right)=(-1)^{k+1} \mathrm{i} \mu_{1, k}-\mu_{2, k} .
\end{gathered}
$$


Since $Q_{k}$ is real,

$$
\operatorname{Im} \operatorname{Res}_{\infty_{k}}\left(z \frac{\psi}{d z}\right)=2(-1)^{k+1} \operatorname{Re} \operatorname{Res}_{\infty_{k}}\left(\phi_{2} \int \phi_{1}\right) .
$$

Equations (20), (21) and (22) prove (19).

\section{A.5 Proof that the equations are independent}

Let $\psi$ be any meromorphic quadratic differential on $\Sigma$ with at most double poles at $\infty_{1}, \cdots, \infty_{M+1}$. In this section we prove that equations (15) to (19) imply that $\psi=0$, provided $t$ is small enough. The idea is to prove that this is true if $t=0$, and conclude by continuity. When $t=0, \Sigma_{t}$ is a noded Riemann surface. In this case, the notion of holomorphic quadratic differential must be replaced by that of a regular quadratic differential : a regular quadratic differential $\psi$ is holomorphic outside the nodes (with at most double poles at $\infty_{1}, \cdots, \infty_{M+1}$ ), and has at most double poles at each side $a_{k, i}^{-}$and $a_{k, i}^{+}$ of each node, with the same residue. (The residue of a $q$-differential at a pole $p$ is the coefficient of $\zeta^{-q}$ in the expansion of $\psi$ in term of a local coordinate $\zeta$ such that $\zeta(p)=0$. This is independent of the choice of the local coordinate). The space of regular quadratic differentials on $\Sigma_{t}$ depends holomorphically on $t$, including at $t=0$.

Let $\psi$ be a regular quadratic differential on $\Sigma_{0}$ satisfying equations (15) to (19). Since $\psi$ has at most double poles at $\infty_{1}, \cdots, \infty_{M+1}, \psi / d z$ is holomorphic at $\infty_{1}, \cdots, \infty_{k}$. Equation (15) imply that $\psi / d z$ has at most simple poles at all $a_{k, i}^{+}, a_{k, i}^{-}$. Equations (16) and (17) imply that the only possible poles of $\psi / d z$ are at $a_{1,1}^{-}, a_{1,2}^{-}$, and $a_{k, 1}^{+}$for $k=1, \cdots, M$. Since $\psi / d z$ has at most one simple pole in each $\mathbb{C}_{k} \cup\left\{\infty_{k}\right\}, k \geq 2$, we conclude that $\psi=0$ in each $\mathbb{C}_{k}, k \geq 2$. Equations (18) and (19) imply that

$$
a_{1,1}^{-} \operatorname{Res}_{a_{1,1}^{-}} \frac{\psi}{d z}+a_{1,2}^{-} \operatorname{Res}_{a_{1,2}^{-}} \frac{\psi}{d z}=0 .
$$

The Residue Theorem in $\mathbb{C}_{1}$ gives

$$
\operatorname{Res}_{a_{1,1}^{-}} \frac{\psi}{d z}+\operatorname{Res}_{a_{1,2}^{-}} \frac{\psi}{d z}=0 .
$$

These two equations imply that $\psi / d z$ has no residue in $\mathbb{C}_{1}$, so $\psi=0$.

\section{References}

[1] C. Costa. Example of a complete minimal immersion in $\mathbb{R}^{3}$ of genus one and three embedded ends. Bull. Soc. Bras. Mat., 15:47-54, 1984.

[2] C. Costa. Uniqueness of minimal surfaces embedded in $\mathbb{R}^{3}$ with total

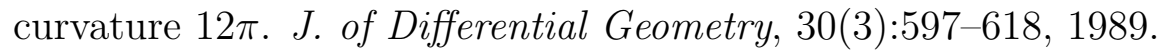


[3] P. Griffiths and J. Harris. Principles of Algebraic Geometry. (Pure and Applied Mathematics). Wiley-Interscience, 1978. MR0507725, Zbl 408.14001.

[4] D. Hoffman and W. H. Meeks III. Embedded minimal surfaces of finite topology. Annals of Math., 131:1-34, 1990.

[5] N. Kapouleas. Complete embedded minimal surfaces of finite total curvature. J. Differential Geom., 47(1):95-169, 1997.

[6] M. Traizet. An embedded minimal surface with no symmetries. J. Differential Geometry, 60(1):103-153, 2002. MR1924593.

[7] M. Traizet. On the genus of triply periodic minimal surfaces. to appear in Journal of Diff. Geom., 2006.

[8] M. Weber and M. Wolf. Teichmuller theory and handle addition for minimal surfaces. Annals of Math., 156:713-795, 2002.

Martin Traizet

Département de Mathématiques et Physique Théorique

Fédération Denis Poisson

Université de Tours

37200 Tours, France

e-mail : martin.traizet@lmpt.univ-tours.fr 